\title{
CRPS: Early Lumbar Sympathetic Block is Better Compared to Other Interventions"
}

\section{TO THE EDITOR:}

“Lumbar Sympathetic Block with Botulinum Toxin Type B for Complex Regional Pain Syndrome: A Case Study" Choi E, Cho CW, Kim HY, Lee PB, Nahm FS.

We read with great interest the recent work by Choi $E$ and colleagues (1) in which they report the use of lumbar sympathetic block (LSB) with botulinum toxin for treatment of complex regional pain syndrome (CRPS) in 2 patients.

However, there are a few points we would like to highlight. Firstly, both the patients were diagnosed to have CRPS on the basis of Budapest criteria which has a specificity of 0.68 and sensitivity of 0.99 (2). Diagnosis was also well substantiated with diagnostic modalities such as 3 phase bone scintigraphy and thermography. Despite the established diagnosis by 4 different tests, the patients were administered a caudal and interlaminar epidural steroid injection.

We would like to suggest that caudal and epidural blocks would have very little pain relieving effect once a diagnosis of CRPS has been established. Moreover, caudal epidural steroid injections are commonly administered to patients with bilateral symptoms, more so in those with radicular or neuropathic symptoms. Hence administering a caudal in the 2 patients mentioned in the report may not be justifiable as they had unilateral symptoms with features highly suggestive of CRPS.

We are of the opinion that LSB could have been administered earlier in the course of treatment, thereby minimizing patient suffering on account of severe pain due to CRPS and the discomfort of repeated interventions.

We further believe the manuscript would have been more valuable to the readers if other medications prescribed for the management of CRPS were also mentioned. However, it is encouraging to notice that early intervention with LSB and botulinum toxin in these patients in the acute phase of disease, led to resolution of CRPS.

\author{
Shikha Awal MD \\ Department of Anaesthesiology \\ Sanjay Gandhi Postgraduate \\ Institute of Medical Sciences, \\ Rae Bareilly Road, Lucknow 226014 \\ Rajashree Madabushi MD \\ Department of Anaesthesiology \\ Sanjay Gandhi Postgraduate \\ Institute of Medical Sciences, \\ Rae Bareilly Road, Lucknow 226014 \\ E-mail: rajashree.mk2@gmail.com \\ Anil Agarwal MD \\ Department of Anaesthesiology \\ Sanjay Gandhi Postgraduate \\ Institute of Medical Sciences, \\ Rae Bareilly Road, Lucknow 226014 \\ Varun Singla MD \\ Department of Anaesthesiology \\ Sanjay Gandhi Postgraduate \\ Institute of Medical Sciences, \\ Rae Bareilly Road, Lucknow 226014
}

\section{References}

1. Choi E, Cho CW, Kim HY, Lee PB, Nahm FS. Lumbar sympathetic block with Botulinum Toxin Type B for complex regional pain syndrome: A case study. Pain Phy- sician 2015; 18:E911-916.

2. Binder A, Schattschneider A, Baron R. Complex regional pain syndrome I (Reflex Sympathetic Dystrophy). In: Wald- man S D. Pain Management. 2nd ed. Elsevier Saunders, Philadelphia 2011, pp 272 $-290$. 